\title{
High-Dose Prednisolone Therapy for Lennox-Gastaut Syndrome Caused by Fentanyl Intoxication-Induced Toxic Leukoencephalopathy
}

\author{
Hui Jin Shin, MD, Ji-Hoon Na, MD, Hyunjoo Lee, MD, Young-Mock Lee, MD \\ Department of Pediatrics, Gangnam Severence Hospital, Yonsei University College of Medicine, Seoul, Korea
}

Received: July 3, 2021

Revised: August 1, 2021

Accepted: August 2, 2021

Corresponding author:

Young-Mock Lee, MD

Department of Pediatrics, Gangnam

Severence Hospital, Yonsei

University College of Medicine, 211

Eonju-ro, Gangnam-gu, Seoul

06273, Korea

Tel: +82-2-2019-3354

Fax: +82-2-2019-4881

E-mail:YMLEEMD@yuhs.ac
Lennox-Gastaut syndrome (LGS) is a severe form of childhood-onset epilepsy characterized by intractable seizures and developmental delay. LGS has a diverse etiology, including genetic, traumatic, metabolic, structural, and autoimmune abnormalities. The optimal therapy for LGS has not been well established, and treatment options generally include anti-epileptic drug therapy; dietary therapy, such as a ketogenic diet; or surgical treatment, such as vagus nerve stimulation, corpus callosotomy, or resective surgery [1]. Steroids have also been shown to be effective in patients with refractory epilepsy, especially in those with features of epileptic encephalopathy, including LGS $[2,3]$. Herein, we report the case of a patient with LGS caused by toxic leukoencephalopathy due to fentanyl intoxication, whose seizure frequency and electroencephalogram (EEG) patterns improved after high-dose steroid treatment.

A 27-month-old boy was diagnosed with toxic leukoencephalopathy caused by oral ingestion of a fentanyl patch at approximately 10 months of age [4]. Fentanyl intoxication may not only lead to cerebral damage and edema, but also cause overall brain damage and neurological deficits in pediatric patients. Toxic leukoencephalopathy was confirmed on brain magnetic resonance im- aging (Fig. 1). The patient's condition led to delayed development and intractable seizures. The patient's age at seizure onset was 11 months. His seizures consisted of his arms alternatively becoming rigid, followed by generalized tonic-clonic movements. The patient was treated with multiple anti-epileptic drugs, including valproic acid, levetiracetam, clobazam, and vigabatrin. Initially, there was an improvement in seizure frequency, but soon after, the seizure frequency increased and the EEG findings continued to deteriorate, leading to the diagnosis of LGS.

Eventually, we decided to proceed with highdose steroid treatment to control the patient's seizures. Prior to the high-dose steroid treatment, the patient experienced traumatic head-drop seizures more than 30 times per day. His EEG results showed a slow and disorganized background rhythm with very frequent multifocal sharp wave discharges, nearly continuous generalized spike and slow-wave discharges (GSSWs), and generalized paroxysmal fast activity (GPFA).

Initially, the patient was prescribed oral prednisolone $(40 \mathrm{mg} /$ day, $2.7 \mathrm{mg} / \mathrm{kg} /$ day) for 7 days. He did not have any serious side effects after steroid treatment. Therefore, we increased the prednisolone dose to $60 \mathrm{mg}$ /day. He was also prescribed a proton pump inhibitor for stress ul-

Copyright (C) 2022 Korean Child Neurology Society

This is an Open Access article distributed under the terms of the Creative Commons Attribution Non-Commercial License (http://creativecommons.org/licenses/by-nc/4.0/) which permits unrestricted non-commercial use, distribution, and reproduction in any medium, provided the original work is properly cited. 

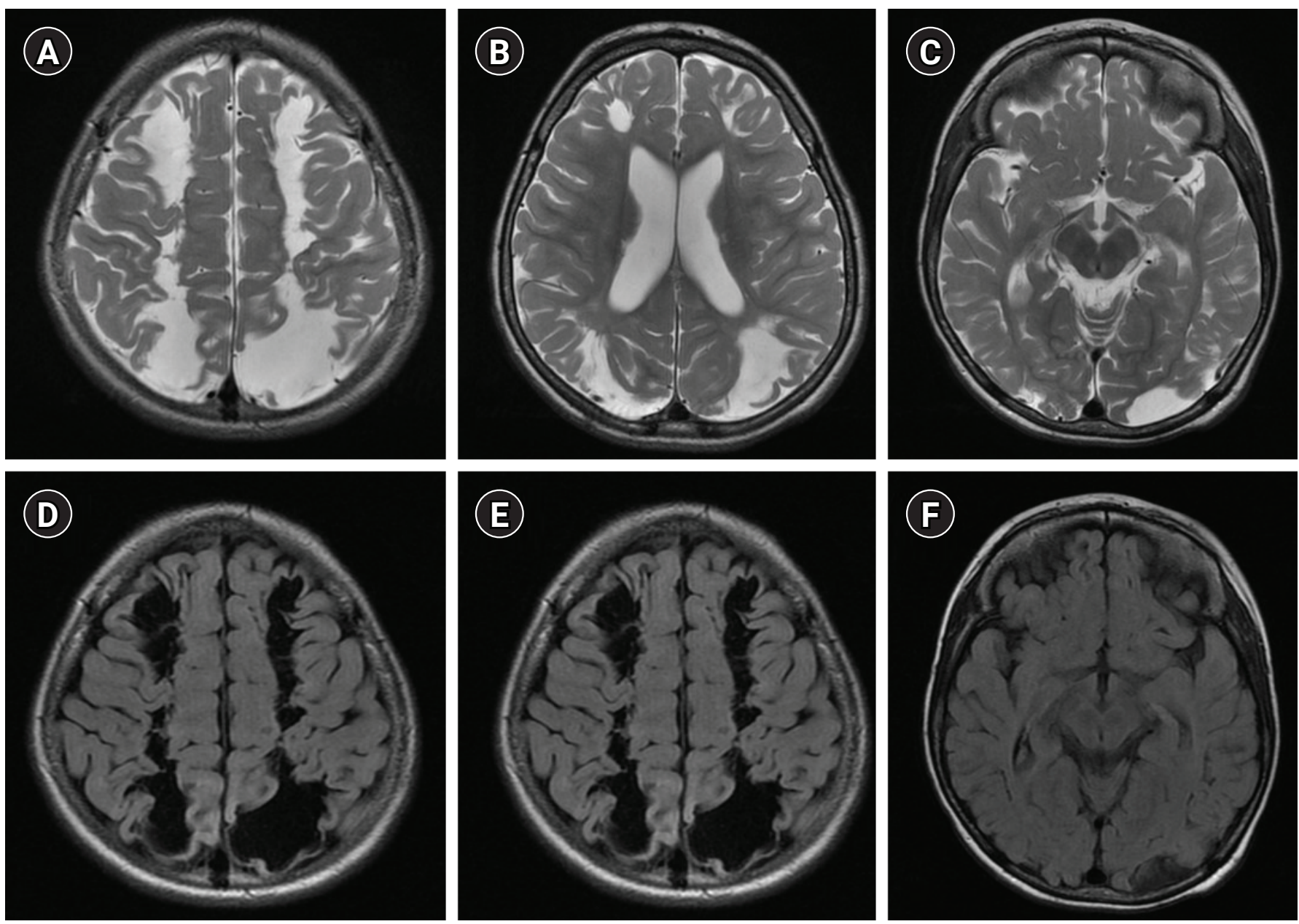

Fig. 1. Magnetic resonance imaging (MRI) findings. MRI findings indicating bilateral cystic encephalomalacia in the two parasagittal cortical border zones and the two frontal lobes. (A, B, C) Axial T2-weighted images. (D, E, F) Axial diffusion-weighted images.

cer prophylaxis to prevent upper gastrointestinal bleeding, which could be caused by high-dose steroids. After 5 days of treatment with the increased dose of prednisolone $(60 \mathrm{mg} /$ day, $4 \mathrm{mg} / \mathrm{kg} /$ day), the patient became seizure-free. On day 7 of treatment with the increased dose of prednisone, the patient's EEG patterns showed a dramatic improvement. His EEG results showed that GSSWs were almost absent, and that the frequency of the brief GPFAs had significantly reduced to less than $5 \%$ (Fig. 2). Since then, the patient has been prescribed prednisolone $(60 \mathrm{mg} /$ day $)$ every other day to reduce the risk of developing Cushing's syndrome, and he has been seizure-free for more than 3 months. The patient has also shown developmental progress since the initiation of prednisolone therapy. We plan to continue the treatment by maintaining high-dose prednisolone $(60 \mathrm{mg} /$ day $)$ every other day for 3 months, and then gradually reducing it over the next 3 months.

This case demonstrates that leukoencephalopathy due to fentan- yl intoxication can progress to LGS, and that high-dose prednisolone therapy may be an effective treatment option for LGS patients with toxic leukoencephalopathy. Although Mitsufuji et al. [5] reported an association between LGS and leukoencephalopathy, the current study is the first to report a case in which LGS associated with toxic leukoencephalopathy was successfully treated. The mechanism by which leukoencephalopathy progresses to LGS remains unknown.

The mechanisms underlying the anti-epileptic effects of steroids also have yet to be elucidated. One hypothesis is that steroids alter neurochemical transmission as a result of alterations in serotonin turnover or gamma-aminobutyric acid uptake. These effects may be mediated through the glucocorticoid or mineralocorticoid receptors, or both [6]. Another hypothesis involves corticotropin-releasing hormone $(\mathrm{CRH})$ and high-dose steroids. High-dose steroid therapy has been used in certain cases of intractable epilepsy, such as West syndrome. Steroid treatment could reduce the levels 


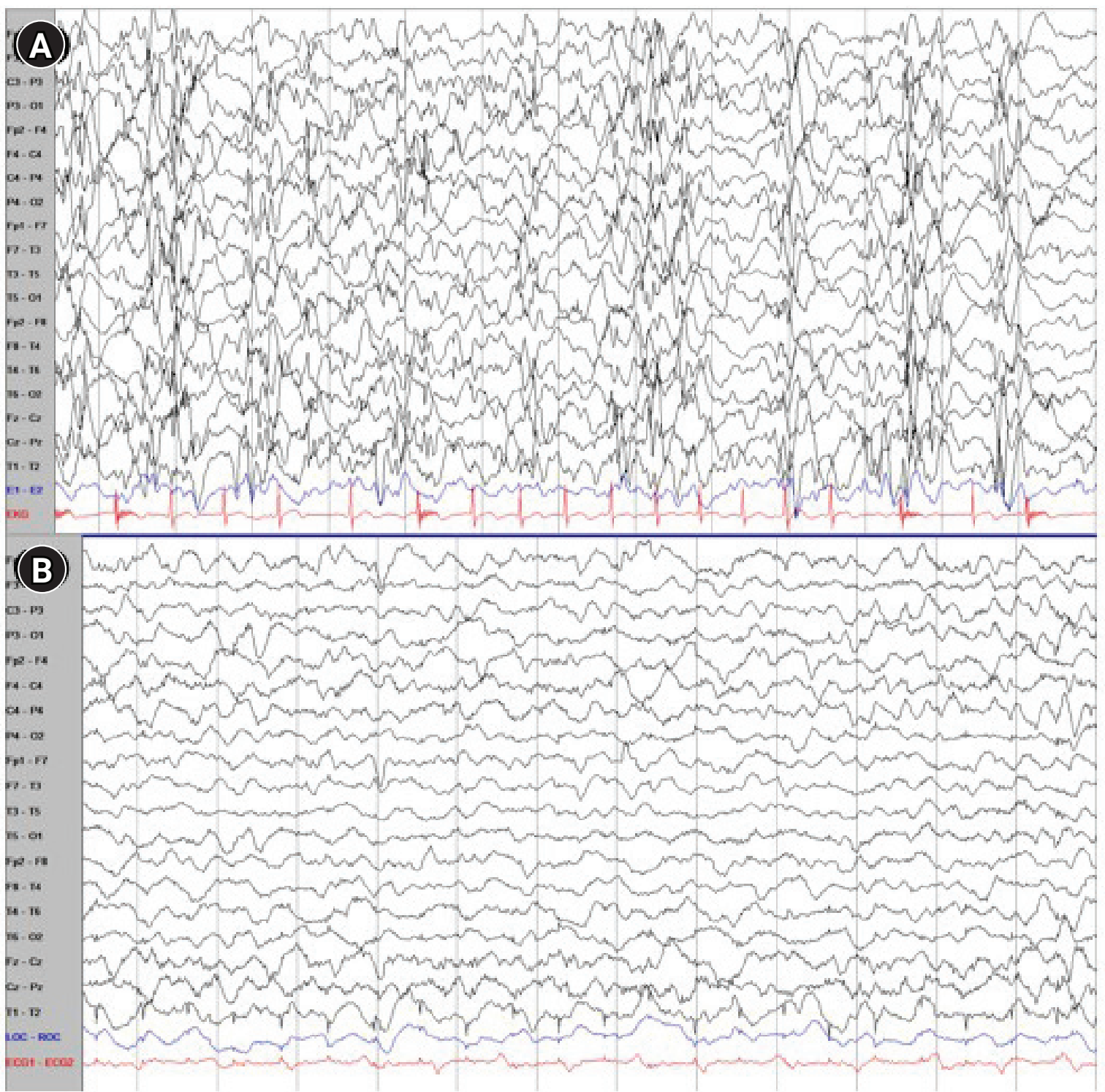

Fig. 2. Electroencephalogram (EEG) findings before and after high-dose steroid therapy. (A) A slow and disorganized background with nearly continuous generalized spike and slow-wave discharges and generalized paroxysmal fast activity (before high-dose prednisolone treatment). (B) A significantly improved EEG status after high-dose prednisolone treatment.

of $\mathrm{CRH}$, a pro-epileptogenic neuropeptide. Another hypothesis is that glucocorticoids may interact with central nervous system steroid receptors, which act as transcriptional regulators that influence voltage-dependent calcium channels [7]. In a previous report on West syndrome, prednisolone was administered orally $(10 \mathrm{mg}$ four times a day) for 2 weeks, accompanied by incremental doses if spasms continued [8].

There are limited studies regarding the use of high-dose steroids in other cases of intractable epilepsy, such as LGS. Therefore, the application of high-dose steroid therapy to treat patients with LGS requires considerable attention, and its efficacy and safety should also be considered. The adverse events associated with predniso- lone therapy include irritability, the development of Cushing's syndrome, electrolyte disturbances, hyperglycemia, glucose intolerance, osteoporosis, bacterial infections, hypertension, and usually reversible dilatation of the ventricular and extra-ventricular cerebrospinal fluid spaces [2]. For this reason, it is important to reduce the dosage of steroids during maintenance and gradually taper it to prevent side effects. The maintenance and tapering methods for high-dose steroids in patients with LGS have not yet been established. It is important to maintain the efficacy of high-dose steroids as much as possible, while minimizing the side effects. Hence, further studies are required to determine the appropriate treatment duration and dosage of high-dose prednisolone therapy. 
In summary, we report that toxic leukoencephalopathy due to fentanyl intoxication can progress to LGS, and that high-dose steroid therapy can be effective in treating LGS in such a situation. This case is expected to be a good reference for pediatric neurologists who treat LGS.

The present study was approved by the Institutional Review Board of Yonsei University Health System (IRB, 3-2020-0358). The board waived the requirement for informed consent.

\section{Conflicts of interest}

No potential conflict of interest relevant to this article was reported.

\section{ORCID}

Hui Jin Shin, https:/ / orcid.org/0000-0002-1541-2564

Young-Mock Lee, https:// orcid.org/0000-0002-5838-249X

\section{Author contribution}

Conceptualization: HJS, JHN, and YML. Data curation: HJS, JHN, and YML. Methodology: HJS, JHN, and YML. Visualization: HJS, JHN, and YML. Writing-original draft: HJS. Writing-review \& editing: HJS, JHN, HL, and YML.

\section{References}

1. Thirunavu V, Du R, Wu JY, Berg AT, Lam SK. The role of surgery in the management of Lennox-Gastaut syndrome: a systematic review and meta-analysis of the clinical evidence. Epilepsia 2021;62:888-907.

2. Bast T, Richter S, Ebinger F, Rating D, Wiemer-Kruel A, Schubert-Bast S. Efficacy and tolerability of methylprednisolone pulse therapy in childhood epilepsies other than infantile spasms. Neuropediatrics 2014;45:378-85.

3. Gupta R, Appleton R. Corticosteroids in the management of the paediatric epilepsies. Arch Dis Child 2005;90:379-84.

4. Lim H, Lee H, Lee YM. Toxic leukoencephalopathy by accidental oral ingestion of an infant's fentanyl patch. Ann Child Neurol 2021;29:93-4.

5. Mitsufuji N, Ikuta H, Yoshioka H, Sawada T. Lennox-Gastaut syndrome associated with leukoencephalopathy. Pediatr Neurol 1996;15:63-5.

6. Mehta V, Ferrie CD, Cross JH, Vadlamani G. Corticosteroids including ACTH for childhood epilepsy other than epileptic spasms. Cochrane Database Syst Rev 2015;2015:CD005222.

7. Jacobson L, Sapolsky R. The role of the hippocampus in feedback regulation of the hypothalamic-pituitary-adrenocortical axis. Endocr Rev 1991;12:118-34.

8. O’Callaghan FJ, Edwards SW, Alber FD, Hancock E, Johnson AL, Kennedy CR, et al. Safety and effectiveness of hormonal treatment versus hormonal treatment with vigabatrin for infantile spasms (ICISS): a randomised, multicentre, open-label trial. Lancet Neurol 2017;16:33-42. 